\title{
Exocytosis Inhibition
}

National Cancer Institute

\section{Source}

National Cancer Institute. Exocytosis Inhibition. NCI Thesaurus. Code C40809.

Exocytosis Inhibition involves interference with, or restraint of, the cellular release of secretory products granules. 